\title{
Deriving Gauge Symmetry and Spontaneous Lorentz Violation
}

\author{
J.L. Chkareuli ${ }^{1}$, C.D. Froggatt ${ }^{2}$ and H.B. Nielsen ${ }^{3}$ \\ ${ }^{1}$ E. Andronikashvili Institute of Physics and \\ I. Chavchavadze Tbilisi State University, 0177 Tbilisi, Georgia \\ ${ }^{2}$ Department of Physics and Astronomy, Glasgow University, \\ Glasgow G12 8QQ, Scotland \\ ${ }^{3}$ Niels Bohr Institute, Blegdamsvej 17-21, DK 2100 Copenhagen, \\ Denmark
}

\begin{abstract}
We consider a class of field theories with a four-vector field $A_{\mu}(x)$ in addition to other fields supplied with a global charge symmetry - theories which have partial gauge symmetry in the sense of only imposing it on those terms in the Lagrangian density which have derivatives as factors in them. We suppose that spontaneous Lorentz invariance breaking occurs in such a theory due to the four-vector field taking a non-zero vacuum expectation value. Under some very mild assumptions, we show that this Lorentz violation is not observable and the whole theory is practically gauge invariant. A very important presupposition for this theorem is that an initial condition is imposed on the no-derivative expressions corresponding to the early Universe being essentially in a vacuum state. This condition then remains true forever and can be interpreted as a gauge constraint. We formulate the conditions under which the spontaneous Lorentz violation becomes observable. Spontaneously broken Lorentz invariance could be seen by some primordially existing or created "fossil" charges with the property of moving through the Universe with a fixed velocity.
\end{abstract}




\section{Introduction}

It is by now a rather old idea to seek to obtain the photon as the Nambu-Goldstone boson for spontaneous Lorentz invariance violation (SLIV) [1]. If, for instance, a four-vector field $A_{\mu}$ takes on in vacuum a non-zero temporally and spatially constant value we have an example of the SLIV [2, 3, 4,. A priori one would then expect in theories with such spontaneous breakdown to see in practice violation of physical Lorentz invariance. It is the purpose of the present article to point out that under some rather mild assumptions, however, one one does not find this breaking of Lorentz invariance to be recognizable experimentally 1 .

A very simple way to achieve the SLIV is to introduce into a usual QED Lagrangian density a potential term $P\left(A^{2}\right)$ for the four-vector field $A_{\mu}(x)$ (so that $\left.A^{2}(x)=A^{\mu}(x) A_{\mu}(x)\right)$ which then after having been transferred to the Hamiltonian density in the usual way could have its minimum for a non-zero value of $A_{\mu}[2$, 4 . In this way a vacuum apparently without Lorentz symmetry can rather easily come about

$$
A_{\mu}(x)=a_{\mu}(x)+n_{\mu} V
$$

where $n_{\mu}$ is an properly oriented unit Lorentz vector with $n^{2}=n_{\mu} n^{\mu}= \pm 1$ for time-like and space-like Lorentz violation, respectively, while $V$ is a proposed SLIV scale.

At first one could easily come to the belief that in such models with SLIV one will find scatterings that indeed reflect the special direction in which the symmetry is broken and thus that one obtains a theory that for all practical purposes breaks Lorentz invariance. However, we deliver a theorem telling that really in a very large class of cases such models do not show Lorentz symmetry breaking but rather behave as an ordinary Lorentz and gauge invariant theory manifesting the SLIV only in some noncovariant gauge choice.

In section 2 we shall put up the type of model which we consider: a rather general type of field theory with a four-vector field $A_{\mu}(x)$ which although it comes to play the role of the four vector potential in electrodynamics is a priori not assumed to obey full gauge invariance - only terms in the Lagrangian with derivatives are assumed to be gauge invariant. And we put forward and prove rather closely related versions of our theorem.

In sections 3 and 4 we argue how it is possible at all to avoid our theorem first by means of initial conditions with what is essentially immovable charges, and then by allowing extra terms in the Lagrangian which give no way to this theorem to work.

In section 5 we make a discussion of that the presuppositions of our theorem especially concerning the need for gauge symmetry of the derivative containing terms

\footnotetext{
${ }^{1}$ Note that previous direct calculations of Lorentz violating processes in the tree $[5]$ and one-loop approximation [6] for QED with the non-linear vector field constraint $A^{2}=V^{2}$ (where $V$ is the SLIV scale, see below), which appear to be completely cancelled, provide explicit examples of this theorem. On the other hand, many counter examples with physical Lorentz violation also exist when underlying assumptions for this theorem are not satisfied 2 , 3].
} 
- are quite reasonable to require in order to avoid bottomlessness of the Hamiltonian and/or to avoid domain walls in cosmology.

Finally, in section 6 we present a resume and conclude.

\section{The theorem}

Now we should have in mind that it is a priori the philosophy of the present article to work with theories that are not a priori gauge invariant. This means that we do not impose gauge invariance on the whole Lagrange density $\mathcal{L}(x)$. Instead, we shall impose gauge symmetry only on the vector field kinetic term or, in general, on the part of our Lagrangian density having derivatives (see for more disussion section 5). Most importantly this means that the kinetic term for the four-vector field $A_{\mu}(x)$ to be the usual one, $-\frac{1}{4} F_{\mu \nu}^{2}$, so as to see that, in spite of that one would have expected to find effects of Lorentz breaking (stemming, say, from some vector field constraint $A^{2}=V^{2}$ where $V$ is the SLIV scale[5, 6]), it turns out to deliver only simple free Maxwell equations. We shall generalize our type of models to include matter fields such as Dirac fields or Weyl fields and a charged scalar that could potentially be used as a Higgs field $\phi$ and also we include into consideration as minimal QED couplings so the non-minimal ones with dimensionful coupling constants. With such matter fields our assumption of the terms with derivatives to be gauge invariant means that most of the terms in the matter field Lagrangian density should actually have the usual gauge invariant form as it takes place for the conventional minimal electrodynamics. However, for the general vector field potential $P\left(A^{2}\right)$ and the non-minimal couplings in the Lagrangian many new gauge breaking terms, including the terms with derivatives, could in principle appear in it through the radiative corrections. We propose that all these terms are very small being substanially suppressed by the high SLIV scale $V$ which is usually thought to be close to the Planck mass $M_{P}$. The simplest choice for possible "large" terms could be then that they were only allowed to depend on the non-derivative Lorentz invariant field combinations from each type of field involved $A^{2},|\phi|^{2}$ and $\bar{\psi} \psi\left(\bar{\psi} \gamma_{5} \psi\right)$ separately but not on their mutual contractions, like as the contraction of the field $A_{\mu}$ with the fermion current $\bar{\psi} \gamma^{\mu} \psi$ apart from its usual occurrence in the minimal QED 3. The complicated high-dimension operators such the four-fermion currentcurrent term etc. are also ignored. This simplest choice for gauge breaking terms is then happened to be a base for the following theorem to work.

Theorem: Consider a Lorentz invariant Abelian field theory with a priori a global charge conservation symmetry only, while gauge symmetry is not imposed in as far

\footnotetext{
${ }^{2}$ Note that minimal (dimensionless coupling) vector-fermion field Lagrangian satisfying the additional global symmetry requirements including charge conjugation, parity reflection and fermion number conservation automtically appears as the conventional QED provided the partial gauge symmetry, namely gauge symmetry of the vector field kinetic term in the Lagrangian is presupposed.

${ }^{3}$ Possible operators of dimension 4 or less include a potential term $P\left(A^{2}\right)$ of fourth order in $A_{\mu}$ and a seagull term $|\phi|^{2} A^{2}$ with an arbitrary coefficient.
} 
we allow for terms containing the squared four vector field $A^{2}$ in combination with globally charge symmetric but derivative free combinations of the matter fields. This means that the theory has in addition to only fully gauge invariant terms - as usual electrodynamics - a (seemingly) gauge breaking term

$$
\mathcal{L}_{b r}\left(A^{2},|\phi|^{2}, \bar{\psi} \psi, \bar{\psi} \gamma_{5} \psi\right)
$$

depending only on the globally phase transformation invariant combinations without any derivatives and only on $A_{\mu}$ via $A^{2}$. Provided now that the Universe should have started with initial conditions so as to ensure in early times the vanishing of the partial derivative of the gauge non-invariant part $\mathcal{L}_{b r}$

$$
\frac{\partial \mathcal{L}_{b r}}{\partial A^{2}}=0
$$

and that $A_{\mu}(x)$ equals a non-zero constant in these asymptotic early times (i.e. a Lorentz symmetry breaking vacuum), then the theory is indeed interpretable as a Lorentz and gauge invariant theory with a non-covariant gauge choice properly fixed in the theory.

The basic idea in the proof of this theorem is that once the Universe gets started in a state in which the "gauge condition" (3) is satisfied, this condition will go on being fulfilled. In fact, we can easily see that by varying $A_{\mu}$ in the whole Lagrangian density, which now is of the form

$$
\mathcal{L}=\mathcal{L}_{i n v}+\mathcal{L}_{b r}
$$

where the terms $\mathcal{L}_{i n v}$ are gauge invariant terms the equation of motion obtained becomes

$$
\partial_{\mu} F^{\mu \nu}=2 A^{\nu} \frac{\partial \mathcal{L}_{b r}}{\partial A^{2}}+j_{\text {matter }}^{\nu} .
$$

Here $j_{\text {matter }}^{\nu}$ is the current coming from the matter fields $\psi$ and $\phi$ other than the four-vector one $A_{\mu}$. With the assumption of requiring global charge conservation - or phase transformation symmetry - for the matter fields we get that the current $j_{\text {matter }}^{\nu}$ becomes conserved $\partial_{\nu} j_{\text {matter }}^{\nu}=0$, and since the lefthand side of the equation (5) is divergence free due to the antisymmetry of $F_{\mu \nu}$, we thus arrive at the conclusion that the vector field current

$$
j_{A}^{\nu}=A^{\nu} \frac{\partial \mathcal{L}_{b r}}{\partial A^{2}}
$$

from the non-gauge invariant term becomes itself separately conserved.

Let us first consider the case of the vacuum background $A^{\nu}$ in the early times were time-like. Then if we start from the mentioned "gauge condition" (3) in early times the conservation of the vector field current $j_{A}^{\nu}$ (6) comes to say that the partial derivative of the gauge non-invariant part of the Lagrangian $\frac{\partial \mathcal{L}_{b r}}{\partial A^{2}}$ does not vary in 
the direction of the four vector field $A_{\nu}$. In fact, the requirement of the separate conservation of $j_{A}^{\mu}$ implies that

$$
\left(\partial_{\mu} A^{\mu}\right) \frac{\partial \mathcal{L}_{b r}}{\partial A^{2}}+A^{\mu} \partial_{\mu} \frac{\partial \mathcal{L}_{b r}}{\partial A^{2}}=0
$$

and thus if $\frac{\partial \mathcal{L}_{b r}}{\partial A^{2}}=0$ at the start, the derivative of $\frac{\partial \mathcal{L}_{b r}}{\partial A^{2}}$ in the direction of the the field $A^{\mu}$, namely the second term in this equation, stays equal to zero. So, this partial derivative $\frac{\partial \mathcal{L}_{b r}}{\partial A^{2}}$ must take the same value all along a curve laid out to follow the $A^{\nu}$ field direction by having these fields as tangents. This means that once it is zero at the beginning it must remain zero along these curves. However, if we start in a vacuum state having Lorentz invariance spontaneously broken through the vector field $A^{\nu}$ vacuum expectation value $\left(<A^{\nu}\right\rangle=n^{\nu} V$ with $n^{2}=1$ for the time-like SLIV), such a beginning with the "gauge condition" (3) fulfilled is basically enforced. One namely then has a non-zero but constant $A^{\nu}$ leading to the $F_{\mu \nu}=0$. Since the matter current $j_{\text {matter }}^{\nu}$ is always zero in vacuum, it follows that also the current $j_{A}^{\nu}=0$.Then, with with $A^{\nu} \neq 0$ we conclude that the factor $\frac{\partial \mathcal{L}_{b r}}{\partial A^{2}}$ in the current $j_{A}^{\nu}$ (6) should also be zero. But once we have $\frac{\partial \mathcal{L}_{b r}}{\partial A^{2}}$ zero at the start, it follows from the equations of motion that this "gauge condition" is satisfied forever.

So we have seen that a very mild initial condition can enforce the special gauge condition (3) and the vanishing of the current $j_{A}^{\nu}$ at all times. Thus the potential possibility for seeing e.g. Coulomb fields in the $F_{\mu \nu}$ around the $j_{A}^{\nu}$ charges, which could reveal the non-gauge invariant properties of the $j_{A}^{\nu}$ current, is in fact prevented. That is to say that an observer, who only has access directly to the usual $F_{\mu \nu}$ fields but not to the $A^{\nu}$ field, could not hope to gain access to the gauge dependent features of $A^{\nu}$ indirectly via the $j_{A}^{\nu}$ anymore, because this current would remain zero.

It should be remarked that for the SLIV to be non-observable we had to assume the special gauge condition (3) as an initial condition, due to the Universe being at first in a vacuum state. Otherwise, by allowing initial deviations from the vacuum state, we would get some true observable breaking of Lorentz invariance. However, such alternative initial conditions means that there are a kind of "fossils" of places where the gauge condition (3) is not fulfilled. Actually such deviations from the vacuum inspired "gauge" would mean that the current $j_{A}^{\nu}$ were not zero but flowed in the direction of the $A^{\nu}$ field. In the coordinate system in which the vacuum $A^{\nu}$ had only the time component $A^{0}$ this would mean "fossil charges" at rest forever. Having such charges at one's disposal the physical Lorentz violation would actually be accessible. In fact the very finding of some "resting" charge of this type, which is only able to follow the direction of the essentially vacuum four vector field $A^{\nu}$, would by itself constitute a strong breaking of Lorentz invariance: these charges do not move at all in the special frame. They could be observed by means of the Coulomb fields, say, by which they would be surrounded. But it should be stressed that we proved above that these fossil resting charges cannot be produced, if the condition (3) is fulfilled all over the Universe and there are no charges of this type to begin with. They can only come from the start, but cannot be produced. 
For simplicity we treated above only the case that the vacuum expectation value of the $A^{\mu}$ field was pointed out in a timelike direction. Actually, we mainly used that case to ensure that the curves having the $A^{\mu}$ as tangents in space-time would come from the past and run into future, so that an assumption about the partial derivative $\frac{\partial \mathcal{L}_{b r}}{\partial A^{2}}$ being zero in the early Universe were sufficient to ensure it once we had proved that it did not vary along these curves. Note however that the frames in which the assumed initial conditions $\frac{\partial \mathcal{L}_{b r}}{\partial A^{2}}=0$ are satisfied actually form a special non-Lorentz invariant set for the case of a space-like $\operatorname{SLIV}\left(<A^{\mu}>=n^{\mu} V\right.$ with $\left.n^{2}=-1\right)$. In such a frame, a curve having a corresponding space-like vacuum expectation value $<A^{\mu}>$ as a tangent will generically have a component along the time direction associated with a non-zero $\left\langle A^{0}\right\rangle$. Thus the proof of our theorem follows in the same way for the space-like as in the time-like case.

\section{Counter examples by initial conditions}

The obvious question to ask is whether we can make the SLIV become observable by not having the initial conditions with $\frac{\partial \mathcal{L}_{b r}}{\partial A^{2}}=0$ which was needed in the theorem. So let us think about rather small deviations from this condition having a perturbatively small $\frac{\partial \mathcal{L}_{b r}}{\partial A^{2}}$. Even in this case the conservation of the current $j_{A}^{\mu}$ following from the basic equation (5) would enforce the partial derivative $\frac{\partial \mathcal{L}_{b r}}{\partial A^{2}}$ to remain approximatively constant along the curves tangential to the $A_{\mu}$ field. So we would indeed find that such perturbatively small deviations from zero would remain constant along these curves. In the approximate vacuum situation with a background vacuum with $A_{\mu} \approx V_{\mu}$ being constant in space-time $\left(V_{\mu}=n_{\mu} V\right.$ where $V$ is a proposed SLIV scale) we could, for instance, in the $V_{\mu}$ timelike case talk about the frame in which the spatial components of $V_{\mu}$ are zero as the frame specified by $V_{\mu}$ and we would simply have the curves tangential to $A^{\mu}$ or approximately to $V^{\mu}$ are the timelike curves meaning the time tracks of resting particles. In this situation the partial derivative $\frac{\partial \mathcal{L}_{b r}}{\partial A^{2}}$ will stay approximately constant as function of time, but could (while we still consider it small) vary as a function of space. In the first approximation we see from the expression (6) that $j_{A}^{\mu}$ now represents a charge distribution $V^{0} \frac{\partial \mathcal{L}_{b r}}{\partial A^{2}}$ which does not change with time. This charge distribution is via the Maxwell equations - our equations of motion derived by the variation of $A_{\mu}$ - observable, as are usual charges, e.g. via the Coulomb field they cause. But now since $A_{\mu}$ or $V_{\mu}$ is neither gauge nor Lorentz invariant in the SLIV case such observation of these fields via the Maxwell equations means that these symmetries are effectively broken and, therefore, SLIV becomes physically observable. Thus our theorem does not work if the initial condition that the partial derivative $\frac{\partial \mathcal{L}_{b r}}{\partial A^{2}}$ be zero is not fulfilled. So, this condition mentioned in the theorem is quite needed.

We should really think of the deviations from zero of this $\frac{\partial \mathcal{L}_{b r}}{\partial A^{2}}$ as representing charge density that must be fossil in the sense that we could not obtain it unless it were there to begin with. One would therefore be reasonable to call the charge density $\rho_{\text {fossil }}=V^{0} \frac{\partial \mathcal{L}_{b r}}{\partial A^{2}}$ a fossil charge.We also remark that it does not change as 
time goes on and thus just rests where it is to begin with. So we could call it the fossil resting charge density. It is just via this resting fossil charge density that the Lorentz non-invariance can come into the theories which in other respects obey the conditions of our theorem but only lacks to obey the initial condition requirements.

One should of course seek experimentally to look for such resting fossil charges. If one had single quanta of them, one might observe them as particles moving with a remarkably fixed velocity. If this velocity were larger than the velocity of an atomic electron, such a fossil charge would produce ionization tracks over long distances in an emulsion without stopping. This would be a characteristic signal and its nonobservation puts a severe upper bound on the density of resting fossil charges with such a velocity. However one might guess that their velocity would follow the cosmic microwave background. In which case the velocity observed in practice would be that of the earth relative to this microwave background, which is of the order of $400 \mathrm{~km} / \mathrm{s}$ [7]. Unfortunately this velocity is too small to produce ionization and the resting fossil charges would not be so easily observed.

In the case of a space-like $\left\langle A^{\mu}\right\rangle$, the fossil charges would look like tachyons passing by. They would then give rise to Cerenkov radiation and would also make tracks in emulsions. The non-observation of such effects again places a severe upper bound on any fossil charge density.

\section{Counter example by allowing extra terms}

The assumptions made in our theorem above might seem a bit arbitrary and not so simple, and indeed are not exceedingly beautiful. Apart from the necessary requirement to have the gauge symmetric $F_{\mu \nu}^{2}$ form for the vector field kinetic terms that we discuss in the next section, we can wonder about why we could not let in the Lagrangian $\mathcal{L}_{b r}$ the "gauge violating " high-dimension operator terms which, for instance, could depend also on a extra construction $A_{\mu} \bar{\psi} \gamma^{\mu} \psi$ etc. in addition to its dependence on $A^{2}, \bar{\psi} \psi$ and $|\phi|^{2}$. The point, however, is that with such a type of term, say $|\phi|^{2} A_{\mu} \bar{\psi} \gamma^{\mu} \psi$, would indeed lead to a model in which the SLIV were observable - of course only through the non-renormalizable terms that now had to be used. Nevertheless, it would in this case be a genuine Lorentz non-invariant theory to live in, even if one starts with the above SLIV vacuum. We can indeed see that if we allow the $\mathcal{L}_{b r}$ to depend also on $A^{\mu} \bar{\psi} \gamma_{\mu} \psi$ so as to become a function of the type $\mathcal{L}_{b r}\left(A^{2}, A^{\mu} \bar{\psi} \gamma_{\mu} \psi, \bar{\psi} \psi,|\phi|^{2}\right)$ we get the new current $j_{A}^{\mu}$ coming from the variation of $A^{\mu}$ in the gauge symmetry breaking part $\mathcal{L}_{b r}$

$$
j_{A}^{\mu}=A^{\mu} \frac{\partial \mathcal{L}_{b r}}{\partial A^{2}}+\bar{\psi} \gamma^{\mu} \psi \frac{\partial \mathcal{L}_{b r}}{\partial\left(A^{\nu} \bar{\psi} \gamma_{\nu} \psi\right)}
$$

and thus the requirement of it being divergenceless $\partial_{\mu} j_{A}^{\mu}=0$ would now no longer immediately lead to that the partial derivative $\frac{\partial \mathcal{L}_{b r}}{\partial A^{2}}$ would stay zero along curves tangentially following the $A^{\mu}$ field even at first being zero at such a curve in the 
early times. Rather we now have an extra term

$$
\bar{\psi} \gamma^{\mu} \psi \frac{\partial \mathcal{L}_{b r}}{\partial\left(A^{\nu} \bar{\psi} \gamma_{\nu} \psi\right)}
$$

in the current $j_{A}^{\mu}(8)$ and when we require its divergenceless $\partial_{\mu} j_{A}^{\mu}=0$ we get the divergence of this extra term into the equation then obtained

$$
\left(\partial_{\mu} A^{\mu}\right) \frac{\partial \mathcal{L}_{b r}}{\partial A^{2}}+A^{\mu} \partial_{\mu} \frac{\partial \mathcal{L}_{b r}}{\partial A^{2}}+\bar{\psi} \gamma^{\mu} \psi \partial_{\mu} \frac{\partial \mathcal{L}_{b r}}{\partial\left(\bar{\psi} \gamma_{\nu} \psi A^{\nu}\right)}+\left(\partial_{\mu} \bar{\psi} \gamma^{\mu} \psi\right) \frac{\partial \mathcal{L}_{b r}}{\partial\left(\bar{\psi} \gamma^{\nu} \psi A_{\nu}\right)}=0
$$

This means that even if we started with the $\frac{\partial \mathcal{L}_{b r}}{\partial A^{2}}$ equal to zero it would not stay zero because of the third term in the equation (10)

$$
\bar{\psi} \gamma^{\mu} \psi \partial_{\mu} \frac{\partial \mathcal{L}_{b r}}{\partial\left(\bar{\psi} \gamma_{\nu} \psi A^{\nu}\right)}
$$

while the last term in it vanishes because the fermion current $\bar{\psi} \gamma^{\mu} \psi$ is conserved. However, provided we arrange for a fermion current flow in the direction of a nonzero gradient multiplier being in the term (11), then the $\frac{\partial \mathcal{L}_{b r}}{\partial A^{2}}$ will vary even if it were initially zero. In fact, since the term $\left(\partial_{\mu} A^{\mu}\right) \frac{\partial \mathcal{L}_{b r}}{\partial A^{2}}$ in the equation (10) is initially zero, there is no way to avoid the variation of the partial derivative $\frac{\partial \mathcal{L}_{b r}}{\partial A^{2}}$ along the curves having the $A^{\mu}$ fields as tangents to be non-zero (see in the above).

Remember that we think of deviations of the $\frac{\partial \mathcal{L}_{b r}}{\partial A^{2}}$ from zero as representing "fossil" charge. Thus, in the above case, fossil resting charge can indeed be produced by a suitable field configuration even if there were no fossil charges to begin with. So, with the extra dependence of the $\mathcal{L}_{b r}$ on the $\bar{\psi} \gamma^{\mu} \psi A_{\mu}$, the SLIV should in principle be observable even in a world that had started out without any so-called fossil charges.

\section{$5 \quad$ Why partial gauge symmetry?}

A crucial assumption in our theorem, that was designated as the partial gauge invariance, is that the kinetic term for the vector field $A_{\mu}$ should take the standard gauge symmetric $F_{\mu \nu}^{2}$ form being also (at least approximately) stable against radiative corrections stemming from the gauge breaking terms $\mathcal{L}_{b r}$ (2). Although we are not able to single out this $F_{\mu \nu}^{2}$ form on any symmetry ground there are two serious arguments in favor of such a choice.

The first argument is related to the physical requirement that the kinetic part of the Hamiltonian should be bounded from below. A priori, one should consider, instead of the $F_{\mu \nu}^{2}$, kinetic terms of the general form, $a\left(\partial_{\mu} A_{\nu}\right)^{2}+b\left(\partial_{\mu} A^{\mu}\right)^{2}$, where $a$ and $b$ are arbitrary constants. Note then that the first term in this form looks like as the kinetic terms of four scalar fields with an exception that the $A_{0}$ component has the "wrong" metric, if we choose $a$ negative so that the spatial components get the right sign for getting positive energy density. So, the $A_{0}$ containing term always gives a negative contribution to the Hamiltonian unless there is a way to get 
rid of the $\partial_{0} A_{0}$ term from the Lagrangian and the spatial derivative on $A_{0}$ terms $\left(\partial_{i} A_{0}\right)^{2}$, which also comes with the "wrong" sign in our term with coefficient $a$. For the momentum $\Pi^{\mu}(x)$ conjugate to $A_{\mu}(x)$,

$$
\Pi^{\mu}=2\left(a \partial^{0} A^{\mu}+b g^{0 \mu} \partial_{\nu} A^{\nu}\right)
$$

the Hamiltonian density becomes in a usual way just

$$
\mathcal{H}(x)=a\left[\left(\partial_{0} A_{\mu}\right)^{2}+\left(\nabla A_{\mu}\right)^{2}\right]+b\left[\left(\partial_{0} A_{0}\right)^{2}-(\nabla \cdot \vec{A})^{2}\right]
$$

which, for $b \neq-a$, can be written in the form

$$
\mathcal{H}(x)=\frac{\left(\Pi_{0}+2 b \nabla \cdot \vec{A}\right)^{2}}{a+b}-a \vec{\Pi}^{2}+a\left(\nabla \cdot A_{0}\right)^{2}-a(\nabla \vec{A})^{2}-b(\nabla \cdot \vec{A})^{2}
$$

So, the only Lorentz covariant way to get rid of the $\left(\partial_{0} A_{0}\right)^{2}$ is to have the equality $b=-a$. Alternatively we can get this kinetic $A_{0}$ term with the right sign by choosing $b$ bigger than the positive $-a$ in the above form. But the gradient energy term from $A_{0}$, i.e. $\left(\nabla A_{0}\right)^{2}$ still would come with the negative coefficient $a$ and thus formally there would be no bottom to the Hamiltonian anyway. Now the equation of motion for $A_{0}$ may be written

$$
(a+b) \partial_{0} \partial^{0} A_{0}-a \triangle A_{0}-b \partial_{0} \nabla \cdot \vec{A}=0
$$

In the limit $a=-b$ this equation reduces to a constraint

$$
-a \triangle A_{0}-b \partial_{0} \nabla \cdot \vec{A}=0
$$

which is in fact the usual Gauss constraint $\nabla \cdot \vec{E}=0$. After partial integration in the action and throwing away boundary terms, this case $a=-b$ leads to the standard kinetic term. Now it is well-known from conventional QED that, if this Gauss constraint is inserted into the Hamiltonian, positivity of the kinetic energy can be guaranteed. However, there is no such constraint available for the general form of the kinetic term. Thus boundedness from below of the Hamiltonian requires a kinetic term of the standard gauge invariant $F_{\mu \nu}^{2}$ form.

There is another, more phenomenological argument for the absence of a gauge non-invariant addition to the vector field kinetic term. This is that the presence of the gauge non-invariant addition in the vector field kinetic term

$$
\mathcal{L}_{\text {kin }}=-\frac{1}{4} F_{\mu \nu}^{2}-\frac{\beta}{2}\left(\partial_{\mu} A^{\mu}\right)^{2}
$$

(where $\beta$ is some positive constant) immediately leads to the domain wall solution for the SLIV, caused by a standard quartic polynomial in $A^{2}$ contained in $\mathcal{L}_{b r}$ (2)

$$
P\left(A^{2}\right)=n^{2} \frac{M^{2}}{2} A_{\mu}^{2}-\frac{\lambda}{4}\left(A_{\mu}^{2}\right)^{2}, M^{2}>0, \quad n^{2}=n_{\mu} n^{\mu}= \pm 1
$$


where the vector field mass squared $n^{2} M^{2}$ may be positive or negative for time-like or space-like Lorentz violation, respectively. Actually, one can find that the vector field $A_{\mu}$ develops a domain wall solutions $V(n \cdot x)$

$$
\begin{gathered}
A_{\mu}=a_{\mu}+n_{\mu} V(n \cdot x), \quad n \cdot x=n_{\mu} x^{\mu} \\
V(n \cdot x)=\sqrt{\frac{M^{2}}{\lambda}} \tanh \left[\frac{(n \cdot x) M}{\sqrt{2 \beta}}\right]
\end{gathered}
$$

with walls being oriented orthogonal to the SLIV direction $n_{\mu}$. Indeed, it is clear that the standard kinetic $F_{\mu \nu}^{2}$ term in the $\mathcal{L}_{k i n}(16)$ is invariant under substitution (18), while the second term in it leads to the differential equation for $V(n \cdot x)$

$$
\beta(\partial \cdot n)^{2} V+M^{2} V-\lambda V^{3}=0
$$

whose solution is just given by Eq.(18). Note that these domain walls are topologically stable since for both time-like SLIV (kink in a time) and space-like SLIV (kink in a preferred space direction) cases the system possesses the disconnected vacua with expectation values $\pm \sqrt{\frac{M^{2}}{\lambda}}$, respectively. Actually, any rotation from one vacuum to another would necessarilly intersect the wall.

These walls could unavoidably lead to a wall-dominated Universe in the early times and, therefore, to its immediate collapse. Thus we have to exclude the presence of the corresponding gauge non-invariant addition to the $F_{\mu \nu}^{2}$ kinetic term (taking constant $\beta=0$ in the $\mathcal{L}_{\text {kin }}(16)$ ) in this case on phenomenological grounds.

\section{Conclusion}

We have discusssed an influence of the spontaneous Lorentz violation on the origin of the gauge internal symmetry in the general Abelian vector field theory. We formulated the theorem which seems to govern this mechanism and found the conditions under which the SLIV becomes physically observable in terms of some primordially existing or created "fossil" charges with the property moving through the Universe with a fixed velocity. The partial gauge symmetry, namely gauge symmetry of the derivative containing terms in the Lagrangian was found to be crucial for our consideration, first of all the gauge symmetry for vector field kinetic term to avoid the botomlessness of the Hamiltonian and exclude the domain wall solution in the SLIV theory that would otherwise lead to the wall-dominated Universe in the early times and, therefore, to its immediate collapse. As a result with gauge invariant kinetic term, one comes in the minimal theory to the exactly QED case with the non-covariant gauge choice as the only SLIV effect.

\section{Acknowledgments}

We would like to thank Rabi Mohapatra for useful discussions. One of us (C.D.F.) wants to thank the Niels Bohr Fund for support to visit Niels Bohr Institute. 


\section{References}

[1] W. Heisenberg, Rev. Mod. Phys. 29 (1957) 269; J.D. Bjorken, Ann. Phys. (N.Y.) 24 (1963) 174; T. Eguchi, Phys.Rev. D 14 (1976) 2755.

[2] V.A. Kostelecky and S. Samuel, Phys. Rev. D 39 (1989) 683; V.A. Kosteletsky and R. Potting, Nucl. Phys. B 359 (1991) 545 (1991); D. Colladay and V. A. Kostelecky, Phys. Rev. D58 (1998) 116002; R. Bluhm and V.A. Kostelecky, Phys. Rev. D 71 (2005) 065008.

[3] S.M. Carroll, G.B. Field and R. Jackiw, Phys.Rev. D41 (1990) 1231; R. Jackiw and V.A. Kostelecky, Phys.Rev.Lett. 82 (1999) 3572; S. Coleman and S.L. Glashow, Phys. Rev. D 59 (1999) 116008.

[4] J.L. Chkareuli, C.D. Froggatt and H.B. Nielsen, Phys. Rev. Lett. 87 (2001) 091601; J.L. Chkareuli, C.D. Froggatt and H.B. Nielsen, Nucl. Phys. B 609 (2001) 46; Per Kraus and E.T. Tomboulis, Phys. Rev. D 66 (2002) 045015; A. Jenkins, Phys. Rev. D 69 (2004) 105007.

[5] Y. Nambu, Progr. Theor. Phys. Suppl. Extra 190 (1968).

[6] J.L. Chkareuli, C.D. Froggatt, R.N. Mohapatra and H.B. Nielsen, hep-th/0412225; A.T. Azatov and J.L. Chkareuli, Phys. Rev. D 73 (2006) 065026.

[7] W.-M. Yao et al. (Particle Data Group), J. Phys. G 33 (2006) 1. 